\title{
RADIATIVE AVALANCHE DRIVEN BY SPHERICAL STARBURSTS
}

\author{
J. FUKUE \\ Astronomical Institute, Osaka Kyoiku University, \\ Asahigaoka, Kashiwara, Osaka 582, Japan \\ M. UMEMURA \\ Center for Computational Physics, University of Tsukuba, \\ Tsukuba, Ibaraki 305, Japan \\ AND \\ S. MINESHIGE \\ Department of Astronomy, Kyoto Univsersity, \\ Sakyo-ku, Kyoto 606-01, Japan
}

As a solid physical mechanism to link between starburst activity and AGNs, we have proposed a radiative avalanche, where the mass accretion is driven by radiation drag exerted by stellar radiation from circumnuclear (ring-like) starburst regions (Umemura et al. 1997a,b). We here examine a radiative avalanche for a case of spherically distributed sources (Fukue et al. 1997). The results of the present spherical case are qualitatively similar to those of the ring-like case. For example, the drift timescale $t_{\text {drift }}$ is found to be $t_{\text {drift }}=$ $1.86 \times 10^{9} \mathrm{yr}(m+1)(b / 100 \mathrm{pc})^{2}\left(L_{*} / 10^{12} L_{\odot}\right)^{-1} g(r)^{-1}$, where $m$ is the power of rotational velocity $\left(v_{\varphi} \propto r^{m}\right), L_{*}$ the starburst luminosity, $b$ the radius of the starburst region, and $g(r)$ a function of the order unity. This timescale is sufficiently shorter than the viscous timescale of the relevant region. In contrast to ring-like starbursts, the advantage of spherical starbursts is the large solid angle subtended by the starburst region. Hence, the luminosity of the starburst region is fully available to avalanche on the surface of the disk. Furthermore, the radiation field of the starburst region has no angular momentum, which is in favor for the avalanche.

\section{References}

Fukue J., Umemura M., Mineshige S. 1997, $P A S J$, in press

Umemura M., Fukue J., Mineshige S. 1997a, ApJL, 479, L97

Umemura M., Fukue J., Mineshige S. 1997b, $A p J$, submitted 